Research Paper

\title{
Predicting Acute Kidney Injury Following Mitral Valve Repair
}

\author{
Chih-Hsiang Chang ${ }^{1,3^{*}}$, Cheng-Chia Lee ${ }^{1,3^{*}}$, Shao-Wei Chen ${ }^{2,3}{ }^{\bowtie}$, Pei-Chun Fan ${ }^{1,3}$, Yung-Chang Chen ${ }^{1}$, \\ Su-Wei Chang 3 , Tien-Hsing Chen ${ }^{4}$, Victor Chien-Chia Wu ${ }^{4}$, Pyng-Jing Lin², Feng-Chun Tsai² \\ 1. Kidney research center, Chang Gung Memorial Hospital, Chang Gung University, College of medicine, Taoyuan, Taiwan \\ 2. Department of Cardiothoracic and Vascular Surgery, Chang Gung Memorial Hospital, Linkou Medical Center, Taoyuan, Taiwan \\ 3. Graduate Institute of Clinical Medical Sciences, College of medicine, Chang Gung University, Taoyuan, Taiwan \\ 4. Department of Cardiology, Chang Gung Memorial Hospital, Linkou Medical Center, Taoyuan, Taiwan \\ * Chih-Hsiang Chang and Cheng-Chia Lee contributed equally to this work \\ $\triangle$ Corresponding author: Shao-Wei Chen, 5 Fu-Shing Street, Kwei-Shan, Taoyuan, Taiwan 333. Tel: 886-3-3281200, Fax: 886-3-3285818, E mail: \\ josephchen0939@yahoo.com.tw
}

( ) Ivyspring International Publisher. Reproduction is permitted for personal, noncommercial use, provided that the article is in whole, unmodified, and properly cited. See http://ivyspring.com/terms for terms and conditions.

Received: 2015.07.15; Accepted: 2015.09.20; Published: 2016.01.01

\begin{abstract}
Background: Acute kidney injury (AKI) after cardiac surgery is associated with short-term and long-term adverse outcomes. Novel biomarkers have been identified for the early detection of AKI; however, examining these in every patient who undergoes cardiac surgery is prohibitively expensive. Society of Thoracic Surgeons (STS) and Age, Creatinine, and Ejection Fraction (ACEF) scores have been proven to predict mortality in bypass surgery. The aim of this study was to determine whether these scores can be used to predict AKI after mitral valve repair.

Materials and Methods: Between January 2010 and December 2013, 196 patients who underwent mitral valve repair were enrolled. The clinical characteristics, outcomes, and scores of prognostic models were collected. The primary outcome was postoperative AKI, defined using the Kidney Disease Improving Global Outcome 2012 clinical practice guidelines for AKI.

Results: A total of 76 patients (38.7\%) developed postoperative AKI. The STS renal failure (AUROC: $0.797, P<.001$ ) and ACEF scores (AUROC: $0.758, P<.001$ ) are both satisfactory tools for predicting all AKI. The STS renal failure score exhibited superior accuracy compared with the ACEF score in predicting AKI stage 2 and 3 . The overall accuracy of both scores was similar for all $A K I$ and $A K I$ stage 2 and 3 when the cut-off points of the STS renal failure and ACEF scores were 2.2 and 1.1 , respectively.

Conclusion: In conclusion, the STS renal failure score can be used to accurately predict stage 2 and $3 \mathrm{AKI}$ after mitral valve repair. The ACEF score is a simple tool with satisfactory power in screening patients at risk of all AKI stages. Additional studies can aim to determine the clinical implications of combining preoperative risk stratification and novel biomarkers.
\end{abstract}

Key words: acute renal failure, cardiothoracic surgery, valvular heart disease, mitral valve repair, patient outcome assessment

\section{Introduction}

Acute kidney injury (AKI) has been associated with increased mortality, length of intensive care unit (ICU) stay, and medical costs after open heart surgery. AKI has been reported in $12 \%-30 \%$ of patients after cardiac surgery. This statistical variation is due largely to differing definitions of AKI and heteroge- neous study populations.[1-4] Postoperative AKI not only increases in-hospital mortality and reduces long-term survival, but results in high medical expenditure, chronic kidney disease, and dialysis dependence.[5-7] Even a minimal increase in the serum creatinine level after coronary artery bypass grafting 
(CABG) is independently associated with increased risk of both long-term mortality and adverse cardiovascular events.[8] In a previous study, $23.8 \%$ of patients undergoing mitral valve intervention experienced AKI following MitraClip implantation.[9] Limited data exist on the occurrence of AKI with conventional mitral repair surgery.

Numerous prognostic risk models for cardiac surgery are currently practiced. Among them, the Society of Thoracic Surgeons (STS) score, published in 2008,[10] is one of the most widely used. The Age, Creatinine, and Ejection Fraction (ACEF) score was first published in 2009 to allow a quick bedside assessment for risk of mortality.[11] Existing risk models for AKI following cardiac surgery focus largely on need for dialysis.[12] In patients who undergo CABG, the STS score is reported to accurately predict the risk of postoperative dialysis.[13] However, a validated score to predict milder AKI not requiring dialysis is lacking. No studies have used these contemporary preoperative risk models to predict the occurrence of AKI and its severity after mitral valve repair. The aim of this investigation was to compare the utility of different scoring systems for predicting postoperative AKI in patients undergoing mitral valve repair.

\section{Material and Methods}

\section{Study design and patient population}

This post hoc analysis of a prospectively collected database was approved by an institutional review board of Chang Gung Memorial Hospital, and the need for individual consent was waived. Between January 2010 and December 2013, the medical records of 312 consecutive patients who received mitral valve repair in a single tertiary referral hospital were reviewed. We excluded patient who had undergone concomitant coronary artery bypass surgery or aortic surgery $(n=95)$, had end-stage renal disease $(n=11)$, had undergone prior cardiac surgery $(n=7)$, had AKI before surgery $(n=2)$, or died on the day of surgery (n $=1$ ). The final cohort comprised a total of 196 patients.

\section{Data collection and definitions}

The clinical characteristics and demographic data of the patients were examined and their STS scores were recalculated using an online calculator. The ACEF score was calculated as age (years)/ejection fraction (\%) +1 (if creatinine $>2.0$ $\mathrm{mg} / \mathrm{dL}$ ).[11] The primary outcome was AKI within 7 days after surgery. The Kidney Disease Improving Global Outcome (KDIGO) 2012 clinical practice guidelines defined AKI as being indicated by an increase in $\mathrm{SCr}$ of $\geq 0.3 \mathrm{mg} / \mathrm{dL}$ within 48 hours or increase in $\mathrm{SCr}$ to $\geq 1.5$ times the baseline within 7 days. Finally, the patients were categorized into 3 severities according to the KDIGO guidelines.[14, 15] A simple model for classifying AKI severity was developed as follows: non-AKI ( 0 points), stage 1 (1 point), stage 2 (2 points), and stage 3 (3 points).[16, 17]

\section{Statistical analysis}

Continuous variables were summarized by mean and standard error unless otherwise stated. The primary end point was the comparison between AKI and non-AKI groups. The Kolmogorov-Smirnov test was used to determine the normal distribution for each variable. The Student $t$ test was used to compare the means of continuous variables and normally distributed data; otherwise, the Mann-Whitney $U$ test was used. Categorical data were tested using the chi-square test or Fisher exact test. Furthermore, discrimination was assessed using the area under the receiver operating characteristic curve (AUROC), which was compared using a nonparametric approach. The AUROC analysis calculated cutoff values, sensitivity, specificity, and overall correctness. Finally, cutoff points were calculated by acquiring the optimal Youden index, defined as sensitivity + specificity -1 , where sensitivity and specificity are calculated as proportions. A $P$ value $<.05$ was considered statistically significant. Overall accuracy was used to evaluate the validity of the screening models.[18]

\section{Results}

\section{Study population characteristics}

We investigated 196 adult patients with a mean age of $57 \pm 1.0$ years, $51 \%$ of whom $(n=100)$ were male. Table 1 lists all patient characteristics. Compared with the patients without AKI, those with AKI were older and more likely to have diabetes mellitus (DM), hypertension, and function class III or IV heart failure. They also exhibited higher levels of serum creatinine and lower levels of albumin and hemoglobin.

Table 2 lists the reasons for mitral valve repair, cardioechograhic results, and preoperative risk scores. Compared with non-AKI, the AKI group had more patients diagnosed with dilated cardiomyopathy. Furthermore, the echograms of the patients with AKI exhibited lower ejection fractions (EFs), higher left ventricular end-systolic diameters (LVESDs), and greater left ventricular end-systolic volumes (LVESVs), indicating long-term left ventricular overload. Unexpectedly, regurgitation severity did not differ significantly between the AKI and non-AKI groups. The mean values of the STS scores for mortality risk and renal failure and ACEF score of all study patients were $4.3 \pm 0.6,4.1 \pm 0.4$, and $1.1 \pm 0.1$, respectively. The observed in-hospital mortality of this study was $5.1 \%$, which was similar to the STS 
score prediction. The STS mortality, renal failure, and ACEF scores differed significantly between the AKI and non-AKI groups.

Table 1. Demographic data and clinical characteristics according to the patients with and without AKI

\begin{tabular}{|c|c|c|c|c|}
\hline & $\begin{array}{l}\text { All Patients } \\
(\mathrm{n}=196)\end{array}$ & $\begin{array}{l}\text { Non AKI } \\
(\mathrm{n}=120)\end{array}$ & $\begin{array}{l}\text { AKI } \\
(n=76)\end{array}$ & $p$-value \\
\hline \multicolumn{5}{|l|}{$\begin{array}{l}\text { Preoperative demographic } \\
\text { data }\end{array}$} \\
\hline Age (years) & $57 \pm 1$ & $52 \pm 1$ & $65 \pm 1$ & $<0.001$ \\
\hline Gender, male (\%) & $100(51.0)$ & $59(49.2)$ & $41(53.9)$ & 0.514 \\
\hline Diabetes mellitus (\%) & $26(13.3)$ & $8(6.7)$ & $18(23.7)$ & 0.001 \\
\hline Hypertension (\%) & $74(37.8)$ & $35(29.2)$ & $39(51.3)$ & 0.002 \\
\hline Pulmonary hypertension (\%) & $124(63.3)$ & $72(60.0)$ & $52(68.4)$ & 0.233 \\
\hline COPD $(\%)$ & $3(1.5)$ & $2(1.7)$ & $1(1.3)$ & 0.845 \\
\hline Old CVA (\%) & $22(11.2)$ & $13(10.8)$ & $9(11.8)$ & 0.827 \\
\hline CHF Fc III /IV (\%) & $92(49.6)$ & $49(40.8)$ & $43(56.6)$ & 0.031 \\
\hline Atrial fibrillation (\%) & $91(46.4)$ & $50(41.7)$ & $41(53.9)$ & 0.093 \\
\hline Mechanical ventilation (\%) & $6(3.1)$ & $3(2.5)$ & $3(3.9)$ & 0.567 \\
\hline $\begin{array}{l}\text { Mean arterial pressure } \\
(\mathrm{mmHg})\end{array}$ & $89 \pm 1$ & $89 \pm 1$ & $89 \pm 1$ & 0.874 \\
\hline ALT (units/L) & $29 \pm 3$ & $28 \pm 3$ & $30 \pm 6$ & 0.581 \\
\hline Serum Creatinine (mg/dL) & $0.9 \pm 0.1$ & $0.7 \pm 0.0$ & $1.1 \pm 0.1$ & $<0.001$ \\
\hline Albumin $(\mathrm{g} / \mathrm{L})$ & $3.9 \pm 0.0$ & $3.9 \pm 0.1$ & $3.7 \pm 0.1$ & 0.015 \\
\hline Hemoglobin (g/dL) & $12.6 \pm 0.1$ & $12.9 \pm 0.2$ & $12.0 \pm 0.2$ & 0.001 \\
\hline \multicolumn{5}{|c|}{$\begin{array}{l}\text { Abbreviation: } \mathrm{AKI} \text {, acute kidney injury; } \mathrm{ALT} \text {, alanine transaminase; } \mathrm{CHF} F \mathrm{Fc} \text {, con- } \\
\text { gestive heart failure functional class; } \mathrm{COPD} \text {, chronic obstructive pulmonary dis- } \\
\text { ease; CVA, cerebral vascular accident. }\end{array}$} \\
\hline
\end{tabular}

Table 2. Comparison of mitral valve pathology, cardioechographic parameters and pre-operative scores of patients with and without AKI

\begin{tabular}{|c|c|c|c|c|}
\hline & $\begin{array}{l}\text { All Patients } \\
(\mathrm{n}=196)\end{array}$ & $\begin{array}{l}\text { Non AKI } \\
(n=120)\end{array}$ & $\begin{array}{l}\text { AKI } \\
(n=76)\end{array}$ & $p$-value \\
\hline \multicolumn{5}{|l|}{ Mitral valve pathology } \\
\hline Rheumatic & 35 (17.9) & $22(18.3)$ & $13(17.1)$ & 0.827 \\
\hline Degeneration & $88(44.9)$ & $60(50.0)$ & $28(36.8)$ & 0.071 \\
\hline Infective Endocarditis & $25(12.8)$ & $17(14.2)$ & $8(10.5)$ & 0.457 \\
\hline Dilated cardiomyopathy & $40(20.4)$ & $14(11.7)$ & $26(34.2)$ & $<0.001$ \\
\hline Others & $8(4.1)$ & $7(5.8)$ & $1(1.3)$ & 0.119 \\
\hline \multicolumn{5}{|l|}{ Pre-operative echogram } \\
\hline Ejection fraction (\%) & $62 \pm 1$ & $64 \pm 1$ & $58 \pm 2$ & 0.022 \\
\hline $\operatorname{LVESD}(\mathrm{mm})$ & $38 \pm 1$ & $36 \pm 1$ & $40 \pm 2$ & 0.031 \\
\hline $\operatorname{LVESV}\left(\mathrm{mm}^{3}\right)$ & $63 \pm 4$ & $54 \pm 4$ & $77 \pm 9$ & 0.016 \\
\hline LVEDD (mm) & $57 \pm 1$ & $57 \pm 1$ & $57 \pm 1$ & 0.918 \\
\hline $\operatorname{LVEDV}\left(\mathrm{mm}^{3}\right)$ & $163 \pm 9$ & $155 \pm 8$ & $174 \pm 10$ & 0.115 \\
\hline Tricuspid Regurgitation III/IV & $26(13.3)$ & $12(10.0)$ & $14(18.4)$ & 0.129 \\
\hline \multicolumn{5}{|l|}{ Pre-operative scores } \\
\hline STS risk of mortality & $4.3 \pm 0.6$ & $2.8 \pm 0.6$ & $6.5 \pm 1.2$ & 0.007 \\
\hline STS renal failure & $4.1 \pm 0.4$ & $2.6 \pm 0.3$ & $6.5 \pm 0.9$ & $<0.001$ \\
\hline $\mathrm{ACEF}$ & $1.1 \pm 0.1$ & $0.9 \pm 0$ & $1.3 \pm 0.1$ & $<0.001$ \\
\hline
\end{tabular}

Abbreviation: ACEF, age, creatinine, and ejection fraction; $\mathrm{AKI}$, acute kidney injury; LVESD, left ventricular end-systolic diameter; LVESV, left ventricular end-systolic volume; LVEDD, left ventricular end-diastolic diameter; LVEDV, left ventricular end-diastolic volume; STS: Society of Thoracic Surgeons.

Continuous variables are presented as mean \pm standard error, and categorical variables, as number (percentage).
Table 3 lists the surgical details and postoperative outcomes. The patients with AKI had a longer bypass time and more tricuspid valve (TV) repair than did the patients without AKI. However, no significant difference in aortic clamp time, maze procedure, or TV replacement was observed. The patients with AKI had longer ICU stays and ventilator time. Of the patients with AKI, 45 were stage 1, 18 were stage 2 , and 13 were stage 3 . Overall, 6 patients received dialysis within 7 days after surgery.

Table 3. Surgical details and postoperative outcomes of patients with and without AKI

\begin{tabular}{|c|c|c|c|c|}
\hline & $\begin{array}{l}\text { All Patients } \\
(\mathrm{n}=196)\end{array}$ & $\begin{array}{l}\text { Non AKI } \\
(\mathrm{n}=120)\end{array}$ & $\begin{array}{l}\text { AKI } \\
(\mathrm{n}=76)\end{array}$ & $p$-value \\
\hline \multicolumn{5}{|l|}{ Surgical detail } \\
\hline Urgent operation, $n$ (\%) & $7(3.6)$ & $4(3.3)$ & $3(3.9)$ & 0.821 \\
\hline $\begin{array}{l}\text { On pump-clamp time } \\
\text { (minutes) }\end{array}$ & $112.9 \pm 3.3$ & $108.4 \pm 3.6$ & $119.9 \pm 6.3$ & 0.117 \\
\hline $\begin{array}{l}\text { On pump-bypass time } \\
\text { (minutes) }\end{array}$ & $166.5 \pm 4.4$ & $156.3 \pm 4.7$ & $182.5 \pm 8.5$ & 0.008 \\
\hline \multicolumn{5}{|l|}{ Concomitant procedures } \\
\hline TV repair & $66(33.7)$ & $30(25.0)$ & $36(47.4)$ & 0.001 \\
\hline TV replacement & $5(2.6)$ & $3(2.5)$ & $2(2.6)$ & 0.955 \\
\hline Maze procedure & $77(39.3)$ & $43(35.8)$ & $34(44.7)$ & 0.214 \\
\hline \multicolumn{5}{|l|}{ Patient outcomes } \\
\hline ICU stay (day) & $4.5 \pm 0.8$ & $3.0 \pm 0.4$ & $6.2 \pm 0.8$ & 0.001 \\
\hline Ventilator duration (hour) & $39.9 \pm 5.5$ & $23.2 \pm 4.5$ & $66.2 \pm 11.7$ & 0.001 \\
\hline AKI stage $1 / 2 / 3$ & - & - & $45 / 18 / 13$ & - \\
\hline $\begin{array}{l}\text { Renal replacement therapy } n \\
(\%)\end{array}$ & $6(3.1)$ & - & $6(7.9)$ & - \\
\hline In hospital mortality n (\%) & $10(5.1)$ & $3(2.5)$ & $7(9.2)$ & 0.037 \\
\hline
\end{tabular}

Abbreviation: AKI, acute kidney injury; ICU, intensive care unit; TV, tricuspid valve

Continuous variables are presented as mean \pm standard error, and categorical variables, as number (percentage)

\section{STS renal failure and ACEF scores in AKI prediction}

The scores were tested for discriminatory power in predicting all AKI, and AKI stage 2 and 3. For all AKI, both scoring systems exhibited satisfactory discriminatory power (AUROC of STS renal failure score: $0.797 \pm 0.032,95 \%$ confidence interval $[\mathrm{CI}]$ 0.733-0.860, AUROC of ACEF score: $0.758 \pm 0.034,95 \%$ CI 0.692-0.825; $P<.001$ ) (Figure 1). For the subgroup analysis, the STS renal failure score showed greater discriminatory power for AKI stage 2 and 3 than did the ACEF score (AUROC $0.835 \pm 0.032,95 \%$ CI $0.773-0.896$ and AUROC $0.747 \pm 0.045,95 \%$ CI $0.659-0.836$, respectively; $P<.001$ ) (Figure 2). To assess the values of selected cutoff points for predicting AKI, the prediction sensitivity, specificity, and overall accuracy were determined (Table 4). The STS renal failure score had a superior Youden index and exhibited the higher overall prediction accuracy compared with the ACEF score in predicting all AKI stages. The STS 
renal failure score also had a superior Youden index but a similar overall accuracy compared with the ACEF score in predicting AKI stage 2 and 3. Compared with the ACEF score, the STS renal failure score was more sensitive (80\% in the all AKI group and $97 \%$ in the stage 2 and 3 AKI groups) but lower specificity in all subgroups.

(a) STS renal failure score

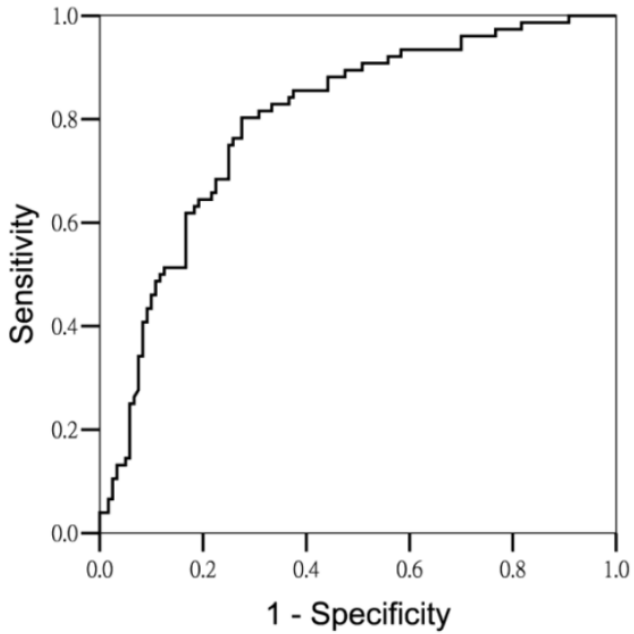

Table 4. Prediction of $A K I$ in different scoring systems

\begin{tabular}{llllll}
\hline Predictive Factors & $\begin{array}{l}\text { Cutoff } \\
\text { Point }\end{array}$ & $\begin{array}{l}\text { Youden } \\
\text { Index }\end{array}$ & $\begin{array}{l}\text { Sensitivity } \\
(\%)\end{array}$ & $\begin{array}{l}\text { Specificity } \\
(\%)\end{array}$ & $\begin{array}{l}\text { Overall Ac- } \\
\text { curacy }\end{array}$ \\
\hline All AKI & & & & & \\
STS renal failure & 2.2 & 0.53 & 80 & 73 & 0.76 \\
ACEF & 1.1 & 0.38 & 54 & 83 & 0.72 \\
AKI stage 2 and 3 & & & & & \\
STS renal failure & 2.2 & 0.62 & 97 & 65 & 0.76 \\
ACEF & 1.1 & 0.42 & 65 & 77 & 0.75 \\
\hline
\end{tabular}

Abbreviation: ACEF, age, creatinine, and ejection fraction; AKI, acute kidney injury; STS, Society of Thoracic Surgeons.

(b) ACEF score

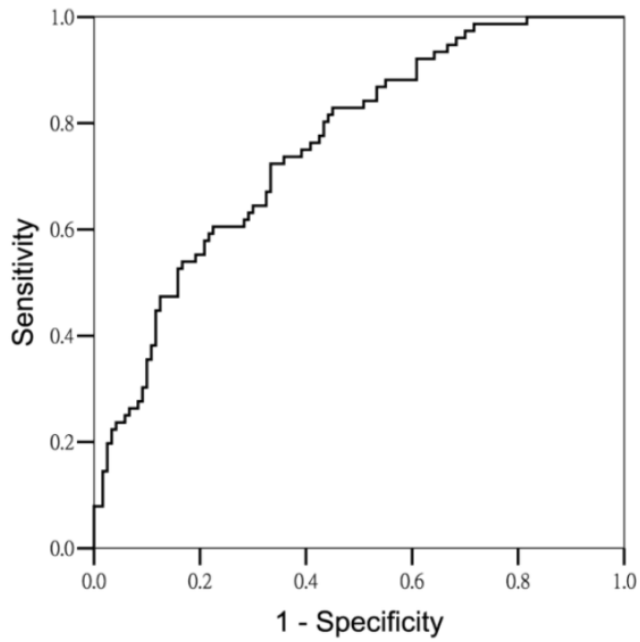

Figure 1. Area under operating curve for all AKI stages according to different predictors. (a) STS renal failure score (AUROC $0.797 \pm 0.032,95 \% \mathrm{Cl}$ $0.733-0.860$ ) (b) ACEF score (AUROC $0.758 \pm 0.034,95 \%$ Cl 0.692-0.825)

(a) STS renal failure score

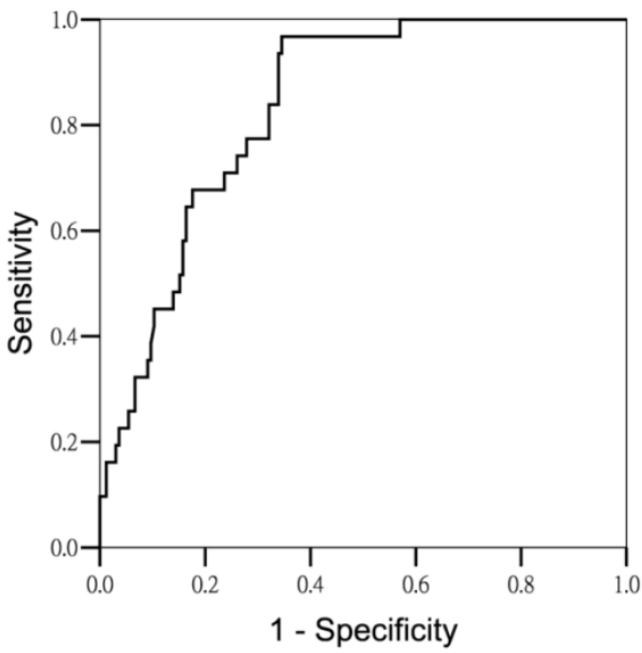

(b) ACEF score

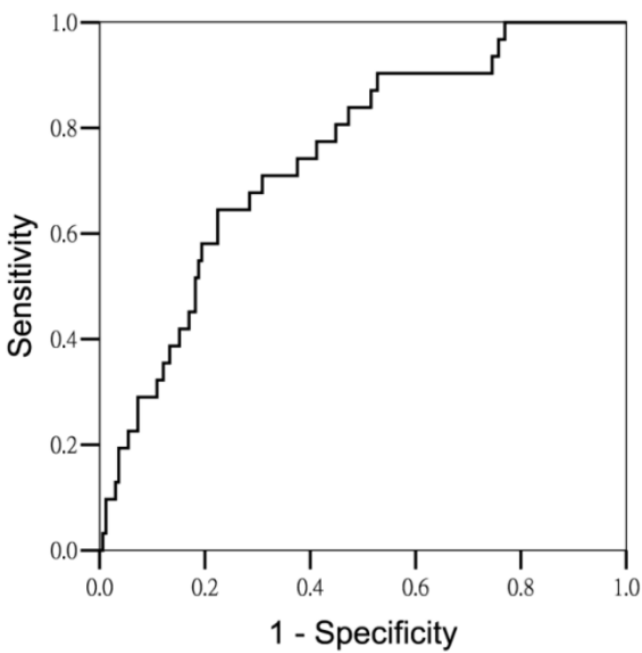

Figure 2. Area under operating curve for AKI stage 2 and 3 according to different predictors. (a) STS renal failure score (AUROC $0.835 \pm 0.032,95 \% \mathrm{Cl}$ $0.773-0.896$ ) (b) ACEF score (AUROC $0.747 \pm 0.045,95 \%$ Cl $0.659-0.836$ )

\section{Discussion}

This study confirmed that the STS renal failure and ACEF scores are both effective tools for predicting postoperative AKI in patients undergoing mitral valve repair. Patients who develop AKI also had longer ICU stays and a higher risk of in-hospital mortality.

Over the past few decades, AKI has been overlooked because of low clinical awareness and the lack 
of an effective method for early detection. AKI development is associated with unfavorable outcomes and high mortality in patients who undergo cardiac surgery. Patients undergoing cardiac surgery typically exhibit complex syndromes with numerous pathways that affect renal function and involve hemodynamic changes, tissue breakdown, reactive oxygen species, infection, and drug toxicity. All of these factors can result in endothelial dysfunction, inflammatory response, and tubular cell damage. AKI following cardiac surgery can be classified as a form of cardiorenal syndrome type 1 , a bidirectional condition that reflects an abrupt decline of renal function secondary to acute cardiac disease or procedures.[19]

Patients who undergo mitral valve surgery typically demonstrate low stroke volume related to mitral regurgitation, which increases the vulnerability of the kidney to injury during cardiac surgery. Our data showed that $38.7 \%$ of the patients who received mitral valve repair had AKI. These patients had more functional class III and IV heart failure, more dilated cardiomyopathy, and lower EFs, which might lead to renal hypoperfusion and higher baseline creatinine. Critically, our data revealed that compared with the non-AKI group, the AKI group exhibited higher rates of DM and hypertension, which are risk factors for occult renal disease. The AKI group also exhibited lower albumin and hemoglobin levels, suggesting the presence of malnutrition because of heart failure. We found that the patients with AKI had larger LVESD and LVESV in cardioechograms. Tribouilloy et al. demonstrated that patients with LVESD $\geq 40$ had unfavorable outcomes and that LVESD is a powerful predictor of survival in patients with organic mitral regurgitation.[20] Although no publication has discussed the association between LVESD and renal dysfunction, suspecting that chronic left ventricular dysfunction indicated by larger LVESD might affect the kidney is reasonable. Furthermore, the AKI group in the current study exhibited longer bypass time and more TV repair, which is consistent with the CABG finding that cardiopulmonary bypass time might be an essential factor for AKI during cardiac surgery.[21] Finally, mortality was higher in the AKI group than in the non-AKI group.

Accurate prediction models can facilitate clinical decision making, patient counseling, and preoperative medical optimization.[22] Currently, the prediction models for severe AKI requiring dialysis are the most robust and externally validated. Among these, the STS renal failure score has exhibited high discrimination in most tested populations.[12] However, because of low dialysis rates $(1 \%-2 \%)$ and late occurrence, the benefits of using these risk models may be limited. More effort is required to develop and vali- date different prediction scores for all AKI severities, particularly because stage 1 AKI is highly common and contributes to numerous short-term and long-term adverse outcomes.[8]

The STS score has been developed and widely used for mortality risk assessment in cardiac surgery. These risk scores are based on a collected database and, by inputting patient demographics and clinical variables into an easy-to-use online calculator, can predict the risk of cardiac surgery mortality. Recently, scoring systems incorporating patient clinical characteristics specific to risk assessment for cardiac surgery have been applied widely to patients undergoing percutaneous coronary intervention. Contemporary preoperative mortality risk scoring systems have also been used to predict major complications such as postoperative AKI.[16] In line with this evidence, Ando et al. demonstrated that the ACEF score can be used to predict AKI in patients undergoing primary percutaneous coronary intervention.[23] This suggests that major postoperative complications or mortality share similar risk factors that may contribute to AKI, such as decreased left ventricular EF and increased serum creatinine level, as we indicated in this study. Collectively, these data confirm the value of using current mortality risk scores to predict AKI following mitral valve repair.

The ACEF score is based on the "law of parsimony" to exclude confounders that might produce bias in the other current scoring system.[11] Incorporating excessive variables results in inaccuracy, severe overfitting, and the risk of multicollinearity. Recent studies found that the ACEF score exhibited similar or superior accuracy compared with previously established scores in stratifying mortality risk in patients undergoing $\mathrm{CABG}[11]$ and percutaneous coronary intervention.[24] This simpler score combines 3 critical clinical natural continuous variable characteristics, namely, age, creatinine (renal insufficiency), and left ventricular EF. Each of these preoperative variables has been clearly demonstrated as independent risk factors for postoperative AKI in cardiac surgery. Therefore, this ACEF scoring system is extremely useful and applicable for predicting postoperative AKI. Furthermore, because calculating the score is quick and easy, the system is appropriate for nonelective surgery. A previous study demonstrated that the performance of the ACEF score in predicting in-hospital mortality in elective and nonelective cardiac surgery is comparable.[25] This finding is consistent with our current results, which suggest that the score has similar applications in predicating all AKI, including for elective and nonelective surgery. Currently, novel biomarkers are used for the early postoperative detection of AKI; however, screening every 
case is not financially feasible. Hence, combining risk assessment and postsurgical biomarkers might improve the cost effectiveness of diagnostic strategies in daily care.

Despite the favorable results obtained in this study, several key limitations should be noted. First, the study was limited by its adoption of post hoc analysis. Second, the sample size was relative small with heterogeneity of etiology and of urgent and elective surgery. Third, because the etiology of AKI is often multifactorial, intraoperative factors and postsurgical care not considered in the scoring systems may have affected the prediction of AKI occurrence. Finally, we did not obtain information regarding mitral valve replacement; the extent of the above-mentioned results in patients with valve replacement should be carefully interpreted.

\section{Conclusions}

To the best of our knowledge, this is the first assessment to compare the prognostic utility of contemporary risk scoring systems for predicting postoperative AKI in mitral valve repair. The STS renal failure score is a more accurate predictor of postoperative stage 2 and 3 AKI. Both the STS renal failure and ACEF scores exhibited satisfactory predictive ability for all AKI severities. However, the ACEF method is simpler and much more user-friendly. We suggest that future biomarker research examine the urine neutrophil gelatinase-associated lipocalin of patients with an STS renal failure score exceeding 2.2 or an ACEF score exceeding 1.1; such research may improve the clinical outcomes of patients with AKI by guiding preventive and early therapeutic strategies. Future studies could also investigate the combination of preoperative risk models and postoperative AKI biomarkers to enhance the prediction accuracy for the future clinical management of AKI.

\section{Acknowledgments}

The authors thank Shu-Yun Wang for assistance in data collection and analysis. This study was partially supported by grants from the Chang Gung Medical Research Fund of Chang Gung Memorial Hospital, Linkou, Taiwan (CMRPG3C1951) and by the Ministry of Science and Technology Research Fund (MOST 103-2314-B-182A-074 and 103-2314-B-182A-018-MY3). The authors also thank all participants from the Kidney Research Center of Chang Gung Memorial Hospital, Linkou, Taiwan.

\section{Competing Interests}

The authors have declared that no competing interest exists.

\section{References}

1. Gallagher S, Jones DA, Lovell MJ, Hassan S, Wragg A, Kapur A, et al. The impact of acute kidney injury on midterm outcomes after coronary artery bypass graft surgery: a matched propensity score analysis. The Journal of thoracic and cardiovascular surgery. 2014; 147: 989-95.

2. Ryden L, Ahnve S, Bell M, Hammar N, Ivert T, Sartipy U, et al. Acute kidney injury after coronary artery bypass grafting and long-term risk of myocardial infarction and death. International journal of cardiology. 2014; 172: 190-5.

3. Rosner MH, Okusa MD. Acute kidney injury associated with cardiac surgery. Clinical journal of the American Society of Nephrology : CJASN. 2006; 1: 19-32.

4. Chen YC, Tsai FC, Chang CH, Lin CY, Jenq CC, Juan KC, et al. Prognosis of patients on extracorporeal membrane oxygenation: the impact of acute kidney injury on mortality. The Annals of thoracic surgery. 2011; 91: 137-42.

5. Parikh CR, Coca SG, Wang Y, Masoudi FA, Krumholz HM. Long-term prognosis of acute kidney injury after acute myocardial infarction. Archives of internal medicine. 2008; 168: 987-95.

6. Chen $\mathrm{TH}$, Chang $\mathrm{CH}$, Lin $\mathrm{CY}$, Jenq $\mathrm{CC}$, Chang MY, Tian YC, et al. Acute kidney injury biomarkers for patients in a coronary care unit: a prospective cohort study. PloS one. 2012; 7: e32328.

7. Lin CY, Tsai FC, Tian YC, Jenq CC, Chen YC, Fang JT, et al. Evaluation of outcome scoring systems for patients on extracorporeal membrane oxygenation. The Annals of thoracic surgery. 2007; 84: 1256-62.

8. Liotta M, Olsson D, Sartipy U, Holzmann MJ. Minimal changes in postoperative creatinine values and early and late mortality and cardiovascular events after coronary artery bypass grafting. The American journal of cardiology. 2014; 113: 70-5.

9. Taramasso M, Latib A, Denti P, Candreva A, Buzzatti N, Giannini F, et al. Acute kidney injury following MitraClip implantation in high risk patients: incidence, predictive factors and prognostic value. International journal of cardiology. 2013; 169: e24-5.

10. O'Brien SM, Shahian DM, Filardo G, Ferraris VA, Haan CK, Rich JB, et al. The Society of Thoracic Surgeons 2008 cardiac surgery risk models: part 2--isolated valve surgery. The Annals of thoracic surgery. 2009; 88: S23-42.

11. Ranucci M, Castelvecchio S, Menicanti L, Frigiola A, Pelissero G. Risk of assessing mortality risk in elective cardiac operations: age, creatinine, ejection fraction, and the law of parsimony. Circulation. 2009; 119: 3053-61.

12. Huen SC, Parikh CR. Predicting acute kidney injury after cardiac surgery: a systematic review. The Annals of thoracic surgery. 2012; 93: 337-47.

13. Mehta RH, Grab JD, O'Brien SM, Bridges CR, Gammie JS, Haan CK, et al. Bedside tool for predicting the risk of postoperative dialysis in patients undergoing cardiac surgery. Circulation. 2006; 114: 2208-16; quiz

14. Palevsky PM, Liu KD, Brophy PD, Chawla LS, Parikh CR, Thakar CV, et al. KDOQI US commentary on the 2012 KDIGO clinical practice guideline for acute kidney injury. American journal of kidney diseases : the official journal of the National Kidney Foundation. 2013; 61: 649-72.

15. Okusa MD, Davenport A. Reading between the (guide)lines--the KDIGO practice guideline on acute kidney injury in the individual patient. Kidney international. 2014; 85: 39-48.

16. Chang $\mathrm{CH}, \mathrm{Fu} \mathrm{CM}$, Yang CH, Fan PC, Li PC, Hsu GY, et al. Society of Thoracic Surgeons score predicts kidney injury in patients not undergoing bypass surgery. The Annals of thoracic surgery. 2015; 99: 123-9.

17. Chang $\mathrm{CH}$, Lin $\mathrm{CY}$, Tian $\mathrm{YC}$, Jenq CC, Chang MY, Chen $\mathrm{YC}$, et al. Acute kidney injury classification: comparison of AKIN and RIFLE criteria. Shock. 2010; 33: 247-52.

18. Alberg AJ, Park JW, Hager BW, Brock MV, Diener-West M. The use of "overall accuracy" to evaluate the validity of screening or diagnostic tests. Journal of general internal medicine. 2004; 19: 460-5.

19. Ronco C, Haapio M, House AA, Anavekar N, Bellomo R. Cardiorenal syndrome. Journal of the American College of Cardiology. 2008; 52: 1527-39.

20. Tribouilloy C, Grigioni F, Avierinos JF, Barbieri A, Rusinaru D, Szymanski C, et al. Survival implication of left ventricular end-systolic diameter in mitral regurgitation due to flail leaflets a long-term follow-up multicenter study. Journal of the American College of Cardiology. 2009; 54: 1961-8.

21. Taniguchi FP, Souza AR, Martins AS. Cardiopulmonary bypass time as a risk factor for acute renal failure. Revista brasileira de cirurgia cardiovascular : orgao oficial da Sociedade Brasileira de Cirurgia Cardiovascular. 2007; 22: 201-5.

22. Thakar CV. Predicting acute kidney injury after cardiac surgery: how to use the "crystal ball". American journal of kidney diseases : the official journal of the National Kidney Foundation. 2010; 56: 605-8.

23. Ando G, Morabito G, de Gregorio C, Trio O, Saporito F, Oreto G. The ACEF score as predictor of acute kidney injury in patients undergoing primary percutaneous coronary intervention. International journal of cardiology. 2013; 168: 4386-7.

24. Wykrzykowska JJ, Garg S, Onuma Y, de Vries T, Goedhart D, Morel MA, et al. Value of age, creatinine, and ejection fraction (ACEF score) in assessing risk in patients undergoing percutaneous coronary interventions in the 'All-Comers' LEADERS trial. Circulation Cardiovascular interventions. 2011; 4: 47-56.

25. Barili F, Pacini D, Rosato F, Roberto M, Battisti A, Grossi C, et al. In-hospital mortality risk assessment in elective and non-elective cardiac surgery: a comparison between EuroSCORE II and age, creatinine, ejection fraction score. European journal of cardio-thoracic surgery : official journal of the European Association for Cardio-thoracic Surgery. 2014; 46: 44-8. 